\title{
L'espion, frère du proscrit. Regards croisés sur la surveillance politique des exilés sous le second Empire
}

\section{Sylvie Aprile}

\section{(2) OpenEdition Journals}

Édition électronique

URL : http://journals.openedition.org/conflits/989

DOI : $10.4000 /$ conflits. 989

ISSN : $1777-5345$

Éditeur :

CCLS - Centre d'études sur les conflits lilberté et sécurité, L'Harmattan

Édition imprimée

Date de publication : 1 mars 2004

Pagination : 9-23

ISBN : 2-7475-6791-5

ISSN : 1157-996X

Référence électronique

Sylvie Aprile, «L'espion, frère du proscrit. Regards croisés sur la surveillance politique des exilés sous le second Empire », Cultures \& Conflits [En ligne], 53 | printemps 2004, mis en ligne le 04 octobre 2004, consulté le 30 mars 2021. URL : http://journals.openedition.org/conflits/989; DOI : https://doi.org/ $10.4000 /$ conflits.989

Ce document a été généré automatiquement le 30 mars 2021

Creative Commons License 


\title{
L'espion, frère du proscrit. Regards croisés sur la surveillance politique des exilés sous le second Empire
}

\author{
Sylvie Aprile
}

1 «Il faut bien le dire, la police de l'exil est faite en majeure partie par les exilés »1.

2 Cette boutade en forme de paradoxe fait l'objet d'un long développement dans l'ouvrage du proscrit Ernest Coeurderoy, Jours d'exil, et n'étonnera guère tous ceux qui ont travaillé sur cette question, mais elle mérite d'être réexaminée et réévaluée dans le contexte français.

3 Elle ne surprend guère car l'étude de la proscription politique sous le second Empire repose en grande part sur les sources de la surveillance politique, ce qui constitue une première forme d'étroite imbrication entre l'exilé et l'espion. Le quotidien et le devenir des exilés passent par le filtre des archives de la police française et des autorités françaises à l'étranger, qui, selon la nécessité du moment, exagèrent ou minimisent l'activité des militants en exil. Ces sources ont ainsi conduit à suivre quelques fils rouges, ceux des conspirations avortées et des sociétés clandestines tout aussi commodes à l'enquête de l'historien qu'au rapport de l'agent de l'Empire.

4 Une plongée dans ces archives pose en réalité plus de questions qu'elle n'apporte de réponses. Dans l'esquisse d'une recherche sur l'exil, Alain Corbin soulignait, il y a quelques années déjà, combien "l'ombre des mouchards et la force avec laquelle leurs rapports pèsent sur la construction des sources risquent de déterminer le tableau brossé par l'historien $»^{2}$. Elles orientent notre regard et « appuient en gras » sur certains visages et conduites. Pour retenir l'attention et être consignée, l'information doit être soit routinière soit sensationnelle.

5 La correspondance diplomatique égrène avec une certaine monotonie deux types d'informations: la première concerne l'embauche du mouchard, la seconde ses rapports. Le recrutement du mouchard se fait, semble-t-il, souvent par une candidature spontanée, qui se monnaye mais se déclare toujours désintéressée. Il faut garder à l'esprit que pour le Consul, la qualité de l'information importe certes, mais elle doit 
surtout être en rapport avec la rémunération négociée. Un rapport de l'Ambassade de France en Angleterre s'achève laconiquement par "A. n'inspire aucune confiance, la somme qui lui est allouée est de 9 livres pour lui et son informateur " ${ }^{3}$. A Londres, le 20 octobre 1852, le représentant français a reçu l'offre de services d'un certain Aubrey, lieutenant colonel des horse guards. Ce dernier prétend tenir ses informations d'un Irlandais qui a fait les campagnes italiennes et qui a déjà servi d'intermédiaire à Mazzini pour se procurer des armes à Birmingham. On le recrute mais sans se leurrer sur la qualité de la marchandise délivrée ensuite. Les attachés d'ambassade ont à l'évidence une certaine répugnance à fréquenter ces personnages douteux et cherchent à éviter un contact direct avec leurs indicateurs qui eux-mêmes expliquent qu'ils doivent rémunérer des informateurs. Le chef de cabinet de l'Ambassadeur écrit à Paris : "J'ai consenti non sans répugnance à écouter ce qu'il pouvait avoir à me dire, il ne m'a fallu qu'un moment pour juger que cet individu ne sait rien de précis et qu'il venait pour offrir ses services moyennant un prix qu'il voulait toucher d'avance $»^{4}$. Aubrey fait état de correspondances par lettres chiffrées et par des avis, insignifiants en apparence, insérés dans les annonces du Times et compréhensibles par les seuls initiés. Pour continuer à percevoir des subsides, Aubrey doit produire des informations nouvelles. Il dénonce ensuite un complot contre la vie du Prince Président, lié à un coup de main prévu sur les frontières, organisé par Pyat et Rybeirolles, deux proscrits, avec le concours de James Fazy ${ }^{5}$. La date n'en est pas encore fixée car selon ses sources on attend le retour de Kossuth qui est alors en tournée aux Etats-Unis. Tout aussi fantasmatique est le réseau d'informateurs qu'il dénonce: la communication entre Paris et Londres serait - toujours selon Aubrey - assurée par des filles publiques. Tous les ingrédients de l'invariant du complot politique sont présents: le caractère international, la complicité d'autorités étrangères et des milieux interlopes ${ }^{6}$.

6 Pour qui a lu ces rapports, c'est peut-être l'imagination déployée par les mouchards, leur capacité à construire puis à dénoncer des réseaux rocambolesques qui font l'essentiel et l'intérêt de ces récits où on ne peut guère relever en définitive d'informations stricto sensu. Comme le note encore Coeurderoy: "Tous les rapports des mouchards sont les mêmes». Il s'amuse déjà des fictions construites par ces pseudo révélations : «Les sociétés de Londres, reliées à celles de Bruxelles et de Jersey se sont mises à la tête de ce mouvement concerté; elles doivent constituer un gouvernement à Boulogne, où elles tenteront une descente prochaine avec les fonds de l'emprunt italien $»^{7}$. Comme pour l'étude de l'état d'esprit public dans les départements, l'utilisation de l'archive soulève de nombreux problèmes : les documents sont rédigés dans le but précis de renseigner le pouvoir central et de lui donner en quelque sorte l'information qu'il souhaite recueillir. Il est donc difficile quoique nécessaire de mesurer l'importance relative des nouvelles et faits saisis. John Merriman donne l'exemple d'un sous-préfet qui écrit au sujet des rapports d'un espion de police envoyé dans l'arrondissement de Tournon « qu'ils ne nous apprennent rien et ne disent que ce j'avais dit moi-même à l'agent $»^{8}$. François Ploux relève aussi la manière dont un événement comme l'attentat d'Orsini majore dans les archives le nombre d'informations concernant d'hypothétiques projets d'assassinat ${ }^{9}$. Les rapports sont en définitive dans les deux cas fort décevants car très répétitifs. On y lit soit une réalité déjà connue et ressassée soit des stratagèmes et des conjectures sans fondements qui ne peuvent nourrir que les représentations que surveillés et surveillants veulent donner d'eux-mêmes.

7 L'information est, quelle que soit sa qualité, discontinue ou du moins limitée dans le temps. En 1857, Thomas Anquetil, réfugié condamné à la déportation en 1850, écrit à 
l'Ambassadeur de France à Bruxelles pour négocier sa remise en liberté. Il se prévaut d'avoir rendu divers services à son pays pendant ses années d'exil. Il a ainsi bénéficié en 1855 d'un sauf conduit puis de l'amnistie, mais il a été à nouveau arrêté ${ }^{10}$. Il donne ensuite des informations très détaillées concernant les livres qui pénètrent en France via le commerce interlope de thé et café, et rédige un mémoire secret sur les imprimeurs clandestins en Belgique. Il dresse également une liste nominative, complétée par des annotations sur les fonds avancés ${ }^{11}$. Le représentant français est ici fort satisfait mais lucide. Il écrit : "On doit utiliser les services de cet homme qui vit dans le besoin au milieu des proscrits $»^{12}$. La qualité du renseignement tient à la condition très difficile de l'espion, qui fait alors preuve d'une grande efficacité. Celle-ci est-elle durable? L'espion disparaît ultérieurement de la correspondance diplomatique. Il faudrait pour chaque cas élucider les modalités de cette disparition.

Les archives des polices étrangères ne montrent également guère d'originalité et il y a peu de dissemblances selon les pays. Leur intérêt pour la proscription peut paraître tout aussi trompeur. On peut avoir l'impression, notamment pour la Belgique, que les autorités sont très vigilantes. Cette vigilance doit être minorée, car il faut replacer ces rapports dans un ensemble plus vaste qui est celui de la surveillance des associations politiques et des mouvements sociaux. Dans ce cadre élargi, on prend la mesure des inquiétudes des autorités belges. Elles sont plus préoccupées par les grèves qui éclatent dans le Borinage que par les agissements des proscrits français. Il faut mettre en parallèle la chronologie et l'intensité des nouvelles traitant des proscrits, et celles des problèmes sociaux et économiques du pays. On peut alors en tirer au moins deux enseignements. La police belge donne par la surveillance politique des gages à la France et la lecture de l'exil ne doit pas être isolée du contexte du pays d'accueil et de la conjoncture diplomatique. En Suisse, la situation varie aussi dans le temps selon les fluctuations de la vie politique locale et surtout des rapports de force entre l'état fédéral et les cantons. La police anglaise, qui se veut une police de maintien de l'ordre et non une police politique, a construit son image par opposition au continent. Alliant la liberté des échanges au droit d'asile, elle récuse toute surveillance mais entretient tout de même un réseau de détectives ${ }^{13}$.

9 Est-ce à dire que cette surveillance n'a de sens que comme histoire de la rumeur et des fausses nouvelles? Non, il ne s'agit pas de nier la dimension ou l'intention répressive des régimes et des pays, mais de voir en elle un moyen d'organisation du champ de la représentation politique. La surveillance dessine des espaces d'inclusion et d'exclusion politiques sur lesquels nous aimerions porter notre attention, tant au fil du temps de l'Empire, que dans des aires géographiques différentes. Notre ambition est de revisiter rapidement cette surveillance en partant, non plus exclusivement des archives d'Etat, mais du regard que portent les exilés eux-mêmes sur ses usages et ses pratiques. La surveillance influe sur la construction narrative et discursive des productions textuelles, qu'il s'agisse des manifestes, de la presse ou de la correspondance. De nombreux écrits en témoignent, rédigés à chaud ou a posteriori. C'est à partir de ces récits, qui mettent en avant quelques figures de proscrits-mouchards, que la proscription se juge et se donne à voir à elle-même. On peut ainsi percevoir comment se construisent les procédés d'identification et les formes de clandestinité. Ses territoires et ses espaces vécus et partagés par les exilés et les espions mettent en évidence la nécessité de l'inter-connaissance et de la fréquentation comme forme de contact politique et de preuve durable de l'engagement. A court et à long terme, se pose 
pour les uns comme pour les autres, les exilés et les espions, la question de l'efficacité de leur action.

Figures d'exilés - figures d'espions

10 La crainte qu'inspire la police impériale n'est pas vaine, et la surveillance policière qui s'exerce ouvertement et clandestinement hors de France est habile et nombreuse. Elle obsède tout autant les proscrits que les autorités impériales, du moins dans les premiers temps. Elle se relâche quelque peu après 1856 mais pour peu de temps puisqu'en 1858, on note à nouveau un regain de surveillance après l'attentat d'Orsini. La méfiance est donc de rigueur et les archives policières françaises et étrangères attestent d'un important réseau d'indicateurs, mais moins visibles et moins spectaculaires que les espions. Les vendeurs de journaux français, des hôteliers à Jersey ou Londres sont les informateurs permanents ou occasionnels des polices. Entre l'espion et les proscrits, il y a toute une trame d'intermédiaires, cafetiers, marchands de tabac qui apparaissent en arrière plan. Ils nous échappent le plus souvent. On en trouve la trace dans le procès Bernard, qui a lieu à Londres, à la suite de l'attentat d'Orsini. Le gouvernement anglais a refusé l'extradition de ce complice d'Orsini, juge Bernard et l'acquitte. La police anglaise a fait venir et payé un certain nombre de personnages chargés de jouer tous ces petits rôles d'intermédiaires. Des garçons de café, des femmes de chambre à Londres constituent l'assemblage complexe des réseaux de comparses.

11 Cette trame rapproche l'espion du mouchard qui fait partie du quotidien des proscrits. Il devient par force un acteur et un témoin de l'existence de l'exil, un de ses familiers.

12 L'importance qui leur est accordée dans les archives du ministère des Affaires étrangères est aussi forte dans les récits d'exilés et leurs correspondances. Elle tient en partie à un passé récent : l'affaire La Hodde, l'agent à la solde de Louis-Philippe, qui a publié ses souvenirs en 1850. Il y montre le rôle joué pendant toute la Monarchie de Juillet par les espions introduits dans les sociétés secrètes. Démasqué par Caussidière, il est convaincu d'avoir joué un rôle nécessaire et explique qu'il a agi comme les chiens de chasse, utiles pour détruire les animaux nuisibles ${ }^{14}$. Les poursuites engagées contre les sociétés secrètes à la fin de la République et tout au long des années 1850 ont encore accru la place accordée à la conjuration et au complot dans l'organisation politique, du côté des conspirateurs ou de ceux qui traquent les républicains.

Sous l'Empire, les exilés ne sont pas entourés de façon imaginaire par des espions et la part de la surveillance policière, à l'intérieur même de la proscription, est estimée jusqu'au quart des exilés. Ils les savent parmi eux et notre connaissance de l'exil passe pour beaucoup par le prisme de l'espionnite. Ces craintes sont constamment évoquées dans les échanges épistolaires. Ces récits n'ont guère intéressé les historiens à la recherche d'informations sur l'action militante et enclins à n'y voir que des propos anecdotiques: "Arthur Lehning lorsqu'il publie une lettre inédite de Déjacque à Pierre Vésinier, ne reproduit pas le passage qui concerne les doutes du premier à l'égard d'un cartain Debuchy $»^{15}$. Lehning écrit : "Ici suit un long passage sur la possibilité d'avoir affaire à un mouchard ». Pourtant ce type d'accusation et la méfiance qui entoure toute nouvelle rencontre sont essentiels car ils conditionnent des pratiques et rites qui font partie du quotidien du politique. Dans un monde où la connaissance ne provient que de la visibilité, il faut faire la preuve de son identité, et c'est parfois un véritable examen de passage qui permet de tester la qualité de proscrit. Dans le journal La Rive Gauche, est publiée le 14 mars 1866 une lettre de Victor Hugo où il se défend contre les accusations 
portées contre lui. Il aurait refusé sa porte à un proscrit. Il déclare : «Il vient à Hauteville House trois à quatre cents visiteurs par an. Il m'est impossible de retenir tous leurs noms. En outre je suis souvent absent. Ce que je puis dire, le voici : quiconque se présente chez moi comme proscrit politique est prié d'en faire la preuve. La moindre suffit mais il en faut une. C'est une habitude dont je ne me départirai pas dans l'intérêt de la dignité de l'exilé " ${ }^{16}$. Même reconnu comme tel, le proscrit doit continuellement réaffirmer cette identité, défendre son honneur contre les rumeurs, les injures et les dénonciations. Ce qui frappe alors, c'est à la fois la juridicisation des pratiques et l'indulgence qui prédomine dans les récits sur le dévoilement de l'espion. Ces pseudos tribunaux sont sous la plume ironique de Coeurderoy des jurys d'honneur. Saint-Ferréol, autre proscrit, les décrit lui comme des tribunaux de famille. Ceci explique que les dépositions et les instructions judiciaires fourmillent dans les récits de la proscription et dans les archives intimes conservées par les exilés. Victor Hugo retrace dans l'Histoire d'un crime, l'affaire Hubert, l'histoire d'un mouchard condamné à mort par les proscrits et sauvé par l'écrivain hostile à un jugement expéditif et à la cruauté de cette peine. La mise en scène de la justice est une nécessité dans un espace sans droit : elle fait du mouchard, un paria, condamné à vivre exclu de la communauté des proscrits ${ }^{17}$. Cette condamnation paraît presque suffisante : le mouchard, l'espion, sont surtout présentés comme des victimes de la misère qu'engendre la proscription. C'est l'apitoiement qui prévaut, même si la fermeté est de règle, car c'est la nécessité qui fait le mouchard et non un revirement politique ou une ambition personnelle ${ }^{18}$. Une affaire d'espion comme l'affaire Schnepp à Genève permet de suivre les multiples réactions qu'elle suscite chez les proscrits et la réflexion qu'elle engendre chez plusieurs d'entre eux sur les mécanismes qui conduisent à trahir ${ }^{19}$. François Joseph Schnepp, âgé alors de 46 ans, né à Hagueneau, coiffeur, a été poursuivi à la suite de la manifestation du 13 juin 1849. Il échappe à son arrestation en s'enfuyant en Suisse. Revenu en France, il est impliqué dans une affaire de société secrète nommée La Nemesis et c'est alors, semble-t-il, qu'il accepte de fournir des renseignements sur ses amis politiques. Il arrive à Berne en novembre 1850 et se présente comme représentant en houblon et en horlogerie puis se rend à Lausanne. C'est là qu'il éveille les soupçons des réfugiés et le 23 novembre, Napoléon Chancel s'introduit dans sa chambre d'hôtel et s'empare de pièces compromettantes. Arrêté le lendemain, interrogé par James Fazy, chef du gouvernement genevois et par l'ancien représentant français et proscrit Beyer, il finit par avouer. Dans le procès-verbal de son interrogatoire, il déclare: "Je reconnais être un agent de la police française, je tiens ma mission d'un individu que je connais sous le nom de Dubreuil et qui demeure rue Jacob». Il raconte ensuite comment il a été amené à se compromettre. Progressivement, presque insensiblement, il a livré des informations de plus en plus précises et préjudiciables pour les siens. Comme le souligne Marc Vuilleumier, qui a relaté cet épisode dans son étude sur les réfugiés français en Suisse, la stratégie employée pour faire de lui un agent est classique, c'est celle qui a été encore utilisée durant la guerre froide: on demande à Schnepp tout d'abord des mémoires très généraux et bien rétribués sur la situation de la presse et les banques agricoles dans le duché de Bade. On flatte ses connaissances -linguistiques ici - pour lui confier ensuite les tâches habituelles du mouchard. Il précise : "Je me suis trouvé invité à des réunions où l'on voulait s'organiser en vue de la résistance. Le lendemain je fus mandé et malgré mon intention de ne rien dire, je fus amené à confesser ce que j'y avais entendu $»^{20}$. Un nouvel élément entre en scène après que l'affaire soit jugée. L'affaire est en effet instrumentalisée par James Fazy pour montrer la collusion des conservateurs avec les autorités françaises et s'en débarrasser au sein 
du conseil genevois. L'acquittement se fait au détriment des conservateurs genevois et accessoirement au profit du proscrit. Schnepp publiera ensuite sa défense.

Outre ces mécanismes intemporels, l'espion apparaît ici comme un autre soi-même de l'exilé. Là se marque peut-être la différence avec les critères relevés par Alain Dewerpe : l'espion est une figure gémellaire, il n'est pas masquée ${ }^{21}$.

Coeurderoy, en retraçant le parcours de Schnepp, détaille le déclassement dont il a été la victime, phénomène qui n'a également guère retenu l'attention des historiens, mais dont on trouve largement la trace dans les dossiers des commissions mixtes ${ }^{22}$. Schnepp fait partie "de ces hommes que la Révolution puis la République ont placé inopinément et sans 'raison' aux côtés d'hommes plus instruits et jouissant d'une certaine notoriété voire notabilité ${ }^{23}$. Cette promotion rapide suivie d'une déchéance tout aussi soudaine, est selon Coeurderoy et la littérature de la proscription, la raison politique de la trahison : "Sortez de pareilles gens de semblables conditions, ils n'ont plus de raison d'être. Leurs grands besoins ne sont plus satisfaits et cependant ils ne peuvent se résigner aux privations. Ce sont des malheureux que la police dispute sans cesse à l'organisation des partis, nouvelle preuve à l'appui de l'utilité de ceux-ci »". "Ils sont», ajoute-t-il, "sur l'étroit sentier qui sépare les deux gouffres de la démagogie et de la police ${ }^{25}$. Coeurderoy remarque ensuite que cette sorte de complicité « contre-nature » est inscrite dans les formes de la socialisation politique en exil. Les espaces privés et publics ne sont pas délimités, le politique n'a pas de lieu précis surtout lorsqu'il doit se maintenir dans une relative clandestinité. Hormis ces transgressions et ces limitations de l'espace public, le temps est aussi un paramètre important. Le mouchard est celui qui, après bien des années, continue à écouter l'exilé, à lui reconnaître une sorte d'identité politique: "L'un est possédé de la manie de la célébrité, et ne peut imaginer de meilleur moyen de réclame que de fournir matière à de nombreux rapports. L'autre croit se donner de l'importance en disant à qui veut l'entendre qu'il médite un régicide. Celui-ci vous annonce confidentiellement qu'avant huit jours il y aura du nouveau. Celui-ci dégonfle son coeur à mesure qu'il vide son verre.(...) Si j'étais ministre de la police, je ferais une rente aux réfugiés $»^{26}$.

Conformisme social et culture politique en partage

Les figures de proscrits qui émergent de ces récits envisagent l'exil dans sa durée et non à ses débuts ou en période de crise, comme le font les archives de la répression. Espion et exilé partagent un quotidien mais aussi une culture politique commune dont les relais sont la rumeur et la fausse nouvelle ; une culture politique reposant sur une morale collective et communautaire qui ne distingue pas le politique du social. Un même conformisme, ou du moins une même vision de la société, s'expriment dans les rapports et dans les récits de l'exil. Les observations données sur les moeurs transposent dans le domaine politique un "normal» et un "pathologique». Les normes sociales influent sur la mise en forme et sur l'activité de la représentation politique de ces groupes.

La condamnation morale qui affecte l'espion vient du fait qu'il apparaît comme un violeur de l'intimité, car il transgresse la frontière entre la sphère publique et la sphère privée. Le souci de l'intime, qui devient plus fort au cours XIXème siècle, est mis en péril dans un milieu qui lui-même est à l'extérieur des lieux légitimes du politique et des rites sociaux. Il y a donc une sorte de fraternité exilés-mouchards dans la perception des modes de transgression. Les agents de police colportent les commérages de la proscription sur l'adultère, la fugue amoureuse, les avances ${ }^{27}$. Le préfet de police Carlier écrit au sujet de Schnepp: "Le sieur Schnepp s'engagea moyennant des 
appointements convenus à fournir au sieur Dubreuil des détails secrets et scandaleux sur ses amis, sur les hommes et les choses de la république sociale $»^{28}$. La presse française se nourrit d'attaques concernant les moeurs de la proscription: "Dubreuil grâce à une foule de renseignements plus ou moins piquants sur les hommes et les choses de la république sociale » a alimenté plusieurs journaux. Le monde de l'exil est un monde qui vit au rythme d'une communauté restreinte et sa respectabilité morale, aussi importante que son exemplarité politique, est mise à mal par les mouchards. La frontière entre le public et le privé est déjà en exil passablement ténue car les bruits et rumeurs circulent vite dans cette collectivité cloisonnée. Le droit au silence et au secret s'oppose à celui de parler et de dire la vérité.

Coeurderoy feint de s'étonner que la maison du mouchard soit identique à celle du proscrit : "Sans répugnance, je me suis assis à sa table, et j'ai couché sous son toit; car il me semblait que la maison de l'espion devait différer des autres. Je m'étais figuré que les murailles étaient sales, les vitres brisées par la foudre $»^{29}$. Le mouchard type n'a pas un déguisement, il ressemble trait pour trait à ses congénères. Sa faiblesse morale découle de son " délabrement physique ", commun à tous les proscrits.

Pour l'exilé, comme pour l'espion, se pose constamment la même question : celle de l'efficacité. La question de l'efficience du mouchard-espion est tout aussi impossible à établir et non moins obsédante que pour le proscrit. Sa situation est également précaire: si l'exilé récuse le temps et surtout le présent, le mouchard n'a guère de chance de vieillir dans cette position qui peut au mieux lui garantir le retour vers la France s'il a été lui-même inquiété. Schnepp selon Coeurderoy déclare : «Au rebours des autres administrations, la préfecture de police confie ses négociations les plus importantes à ses agents les plus nouveaux. On est sitôt connu, on vieillit vite dans la carrière de l'infamie! ${ }^{30}$. Si tous deux ont pour tâche d'informer, cela n'est pas seulement ce qu'on attend implicitement d'eux. Ceci transparaît à travers la figure omniprésente et singulière de John Hitschens Sanders ${ }^{31}$. Cet homme est pourtant étrangement absent des récits des proscrits français et des archives françaises comme s'il semblait établi une fois pour toutes qu'il n'y a pas de police politique anglaise. Il joue pourtant un rôle majeur établissant des rapports sur des réunions des proscrits européens à partir en 1849 et surtout de 1850-51, où il est recruté, semble-t-il lors l'exposition universelle, comme détective constable attaché à la division $\mathrm{A}$ de Whitehall. Il a vécu en France de nombreuses années, parle couramment français et a même un accent français en anglais. Promu sergent en 1851, inspecteur en 1856 à 31 ans, il a un ou deux hommes qui travaillent avec lui. Sanders meurt en 1859 et la Foreign Branch disparait, semble-t-il, avec lui. Sa position est pour le moins très particulière et révélatrice. En premier lieu, elle entre en contradiction avec la philosophie de la Metropolitan police qui récuse, selon ses propres termes, "l'espionnage de personnes dont on n'est pas sûr des intentions délictueuses » et surtout pour des raisons politiques. Les activités de Sanders sont d'ailleurs dénoncées, et dès lors dévoilées durant l'affaire Orsini.

Les rapports de Sanders sont, au-delà de leur existence même, passionnants, non par ce qu'ils nous apprennent sur la proscription étrangère à Londres et à Jersey, mais sur la personnalité même de Sanders ou du moins ce qu'on peut en découvrir. Il apparaît ainsi comme une sorte d'archétype de l'espion exemplaire. Il est une sorte d'hommeorchestre, présent partout où il le faut, vérifiant toute rumeur et collectant tout indice. Cette efficacité se traduit par un regard satisfait qui s'explique par son souci constamment exprimé du travail bien fait. Il en conclut à plusieurs reprises «que rien ne 
peut se passer sans qu'il le sache $\aleph^{32}$. Son attitude prudente et consciencieuse conforte la position britannique qui est de minimiser les actions possibles des réfugiés. Ceci rejoint l'hypothèse développée par Bernard Porter selon laquelle dans le cadre britannique, l'espion n'est pas là pour informer, et pas davantage pour dramatiser, mais au contraire pour rassurer. L'image que donne Sanders de la proscription est celle d'un monde démoralisé, inapte, mais qu'il ne stigmatise pas. Il distingue tout au plus le monde des vrais réfugiés de celui des agents provocateurs, qui sont les plus excités. Il semble très probable que Sanders informe également les proscrits et montre par son existence même la nécessité d'un double jeu, qui peut seul donner une information précise et susceptible de rassurer. Loin de dévaluer ses estimations, le fait qu'il donne un nombre de proscrits inférieur à ce qui est aujourd'hui admis, est le produit de cette ambivalence. On peut décrypter cette pratique grâce aux études faites sur la criminalité en Angleterre. Certes les chiffres donnés par Sanders minimisent le nombre de proscrits, mais plus que de donner un nombre il s'agit pour la police de connaître et de catégoriser.

On peut s'étonner qu'aucun proscrit français n'évoque directement Sanders, alors qu'a contrario, l'exilé russe Herzen consacre au détective et ses sbires quelques pages pleines d'humour, célébrant en quelque sorte les vertus de "l'espionnage de proximité ». Herzen, présent au procès de Simon Bernard, le complice d'Orsini, est étroitement surveillé et encadré par un détective très prévenant: "Sanders, un des chefs de ladetective police, parla tout bas à l'un des siens et lui ordonna de me surveiller, c'est-à-dire me montra tout bonnement du doigt à un détective qui, dès cet instant se trouva toujours dans mes parages. Je ne puis assez exprimer ma gratitude pour ces instructions données par son supérieur: si, pendant le quart d'heure de repos des juges, je m'absentais pour vider un verre d'ale à la taverne, et au retour je ne trouvais pas de place, le policier m'indiquait où je pouvais m'asseoir. Si j'étais arrêté à l'entrée par un de ses collègues, il lui faisait signe et on me laissait passer $\aleph^{33}$.

Cette information nous éclaire sur les formes extrêmement simples et frustes de la surveillance. Cette proximité, cette sollicitude, participent plus globalement à une «mise à distance » de la violence qui est alors en jeu chez les proscrits et ceux qui les surveillent. L'espion et le mouchard ainsi sont liés à une autre figure qui hante la proscription française, celle du tyrannicide décrit ainsi par Hugo: "Un inconnu très mystérieux, vient vous parler bas à l'oreille; il vous déclare que, si vous voulez il se charge d'assassiner l'empereur; c'est Bonaparte qui vous offre de tuer Bonaparte. A vos banquets de fraternité, quelqu'un dans un coin criera: Vive Marat! Vive Hebert! Vive la guillotine! avec un peu d'attention vous reconnaitrez la voix de Carlier $»^{34}$. Le proscrit et l'espion sont là, face à face, pour déjouer le même crime.

Cette crainte n'est pas seulement celle de la place accordée à la violence. Elle est aussi marquée par une obsession : celle de l'inversion des rôles, ici démultipliée. Décrivant le procès fait par les proscrits au mouchard Hubert, Hugo écrit: "Cahaigne, vieux conspirateur déclare à Hubert: 'Nos amis sont dans les mains de Bonaparte mais vous vous êtes dans les nôtres, faites des révélations, éclairez-nous. Aidez-nous à sauver nos amis ou vous êtes perdu. Parlez!' Hubert lui répond: 'C'est vous qui perdez nos amis de Paris en disant leurs noms tout haut dans une assemblée où il y a évidemment des mouchards' ${ }^{35}$.

24 Coeurderoy écrit quant à lui : "J'ai mouchardé Schnepp». Le jeu de miroir est ici implicite. Jugés immoraux et dangereux par la proscription française, ces «hommes doubles » connaissent en comparaison une situation presque licite dans la proscription 
allemande - où Willich devient informateur selon ses dires pour obtenir des informations, où Marx lui-même utilise les services d'un espion pour surveiller les autres exilés allemands ${ }^{36}$. Pour ces proscrits, la trahison semble beaucoup plus logiquement incluse dans le jeu politique. Seul le français Coeurderoy, paria politique lui-même, et l'historiographie étrangère et surtout anglo-saxonne, évoquent cette situation de la proscription où chaque parti espionne l'autre. Doit-on en conclure que l'exemplification vertueuse voulue par les proscrits, que l'art du discours comme l'effet des sources semblent encore établir un miroir déformant pour les historiens français?

En accordant plus d'attention aux exilés comme éléments majeurs de la surveillance politique, non plus victimes ou témoins mais acteurs, on recoupe en définitive les interrogations posées par Nathalie Bayon en introduction à ce numéro, sur la frontière très floue qui sépare les pratiques politiques et policières et qui existe entre l'« agir politique » et l'«agir policier ». La surveillance politique des exilés au XIXème siècle participe à la réglementation progressive des pratiques politiques. Elle sert dès lors à délimiter les lieux dans lesquels cette activité doit prendre place et à circonscrire les groupes admis à y participer. Elle induit aussi un travail d'histoire comparée qui n'est ici qu'à l'état d'ébauche. Par la mise au jour d'analogies et divergences selon les pays et les communautés en exil s'esquissent des discours et des usages politiques sur soi et sur l'autre qui doivent être inclus dans l'élaboration des politiques d'exil.

D'autres comparaisons s'imposent aussi, car dans d'autres lieux comme la prison ou dans le monde de la criminalité par exemple, l'espionnage et le mouchardage constituent également, à des échelles et selon une graduation variables, des instruments majeurs de circulation de l'information. On peut donc y voir non plus un trait spécifique aux territoires du politique mais un phénomène commun à des espaces sociaux ou politiques considérés comme illégitimes.

\section{NOTES}

1. . Coeurderoy E., Jours d'exil, Editions Canevas, 1991, p. 246.

2. . Corbin A., L'exil, Revue d'histoire du XIXème siècle, $\mathrm{n}^{\circ}$ 11, 1996, introduction, p. 13.

3. AMAE, Archives du ministère des Affaires étrangères, Grande-Bretagne. Affaires politiques diverses. 1852. R215.

4. . Ibid.

5. . Né le 12 mai 1794 à Genève où il décède le 6 novembre 1878, James Fazy a été le fondateur en 1825 du Journal de Genève et en 1846 du Parti radical genevois. Entre 1847 et 1860 , il est plusieurs fois Président du Conseil d'Etat de la République et du Canton de Genève.

6. . Sur les rapports entre bohème et conspiration, voir Traverso E., « Bohème, exil, et révolution. Notes sur Marx et Benjamin »,La pensée dispersée, figures de l'exil judéoallemand, éditions Léo Scheer, 2004.

7. . Coeurderoy E., Jours d'exil, op. cit., p. 226. 
8. . Merriman J., "Au temps des sociétés secrètes », Fidélité républicaine et monde rural, 1848-1851, éditions de la société savante d'Auvergne, 2001, p. 304.

9. . Ploux F., De bouche à oreille, naissance et propagation des rumeurs dans la France du XIXème siècle, Aubier, 2003, p. 61. Voir l'intervention de Pierre Karila-Cohen au colloque « Regards croisés entre politistes et historiens sur la surveillance politique » (organisé par le Centre d'Etudes sur les Conflits, au CERI, les 24 et 25 mars 2003) : «L'invention de la police de l'opinion sous la Restauration et la monarchie de Juillet : origines intellectuelles et pratiques administratives ».

10. . AMAE, Belgique 1854-1857-ADP7.

11. Ibid. L'imprimeur Rozez cache ces ouvrages dans le magasin d'indiennes que tient sa femme à Chaerbeck.

12. . Ibid.

13. . Ce qui nous intéresse ici est la façon dont les exilés intériorisent le discours des autorités britanniques. Concernant la police anglaise, des travaux récents nuancent voire remettent en cause l'idée qu'elle puisse être détachée de la surveillance politique. Voir Emsley C., The English Police. A Political and Social History, Hemel Hempstead, Harvester Wheatsheaf, 1991. Rainer R., « La tradition policière britannique : modèle ou mythe ? ", Cahiers de la sécurité intérieure, n7, 1991-1992, pp. 27-39. Je remercie Dominique Kalifa pour ces références sur la police anglaise.

14. . Hodde L. (de la), Histoire des sociétés secrètes et du parti républicain de 1830 à 1848, Paris, Julien, Lanier et Cie, 1850. Cet ouvrage et celui que publie un autre espion, Chenu A., Les Conspirateurs, ont retenu l'attention de Marx et Engels qui en publient un compte rendu dans la Neue Zeitung, le quotidien démocratique de Cologne. Dans leur sillage, ils formulent la première critique marxiste du blanquisme. Enzo Traverso, op. cit., p. 33.

15. . Muller Lehning A., « Une lettre de Joseph Déjacque à Pierre Vesinier », Bulletin of the international institute of social History, ${ }^{\circ} 1,1951$.

16. . La Rive Gauche, 4 mars 1866.

17. Sur l'émergence au XIXème de la figure du paria et sa conceptualisation au cours du XXéme siècle voir Leibovici M. et Varikas E. (dir.), « Le paria, une figure de la modernité ", Tumultes, $n^{\circ}$ 21-22, novembre 2003, pp. 87-107.

18. . Victor Hugo écrit : « En remuant mes papiers, j'y ai trouvé une lettre de Hubert. Il $\mathrm{y}$ a dans cette lettre une phrase triste : 'La faim est mauvaise conseillère'. Hubert a eu faim. ", Hugo V., Choses vues 1849-1885, Hubert Juin éd., Folio-Gallimard, 1972, p. 329. 19. . Vuilleumier M., « La question des réfugiés dans les luttes politiques de Genève entre 1848 et 1857 », Le refuge en Suisse après les révolutions de 1848, Revue des archives fédérales, n²5, éditeurs Verlag Paul Haupt, Berne, 1999, pp. 147-196.

20. . Ibid.

21. . Dewerpe A., Espion , une anthropologie du secret contemporain, Gallimard, 1994, p. 385.

22. . Aprile S., « Coupables d'excitation, Réflexions sur les intermédiaires et médiateurs des luttes républicaines dans les campagnes ", Fidélité républicaine et monde rural, Actes du colloque d'Aurillac, 2001, pp. 309-325.

23. . Coeurderoy E., op. cit., p. 239.

24. . Coeurderoy E., op. cit., p. 239.

25. . Dewerpe A., Espion, op. cit., p. 385.

26. . Ibid., p. 248 . 
27. . Martinez P., Paris Communard, Refugees in Britain (1871-1880), PHD thesis, University of Sussex, 1981, p. 159.

28. . Vuilleumier M., La question des réfugiés dans les luttes politiques de Genève entre 1848 et 1857 (...), op. cit., pp. 147-196.

29. . Coeurderoy E., op.cit., p. 243.

30. . Coeurderoy E., op.cit., p. 243.

31. . Porter B., The refugee Question in Mid-victorian Politics, Cambridge University Press, 1979 , pp. 47-50, pp. 84-85.

32. . Public Records Office, HO 45/846.

33. . Herzen A., Passé et méditations, L'Age d'homme, 1985, tome III-IV, p. 101.

34. . « Quelquefois l'espion mendie ; l'empereur vous demande l'aumône par son Pietri, vous donnez, il rit, gaîté de bourreau. Vous payez les dettes d'auberge de cet exilé, c'est un agent. Coco se déguise en Sceavola Victor Hugo, Pendant l'exil, Actes et paroles, Hetzel, pp. 7-8.

35. . Hugo V., Choses vues, 1830-1871, La Palatine, Genève, 1944, pp. 472-473.

Un des « juges », Fillion déclare : « En Afrique nous avons eu une affaire comme celle-là. On a découvert qu'un nommé Auguste Thomas était agent de police, un ancien républicain pourtant, et de la veille et de toutes les conspirations depuis vingt ans. On a eu la preuve du fait un jour, à neuf heures du soir. Le lendemain, l'homme avait disparu sans qu'on ait pu jamais savoir ce qu'il était devenu. C'est comme cela que les choses se font ». Hugo V., Choses vues, op. cit., p. 477.

36. . Latteck C., " German socialism in London after 1849, the communist league of August Willich and Karl Schopper ", Exiles from european revolution, edited by Sabine Freitag, Berghahn press, 2003, pp. 187-209.

INDEX

Index géographique : France

Index chronologique : 19e siècle

Mots-clés : histoire, police, Renseignement, surveillance 